\begin{tabular}{|c|l|}
\hline Title & $\begin{array}{l}\text { THE LOCAL MODEL OF AN ISOTROPIC MAP-GERM ARISING FROM ONE-DIMENSIONAL SY MPLECTIC } \\
\text { REDUCTION }\end{array}$ \\
\hline Author(s) & ISHIKA WA, G. \\
\hline Citation & MATHEMATICAL PROCEEDINGS OF THE CA MBRIDGE PHILOSOPHICAL SOCIETY, 111, 103 112 \\
\hline Issue Date & 1992 \\
\hline Doc URL & http://hdl.handle.net/2115/7397 \\
\hline Rights & Copyright $\odot 1992$ Cambridge University Press \\
\hline Type & article \\
\hline File Information & MPCPS111.pdf \\
\hline
\end{tabular}

Instructions for use 


\title{
The local model of an isotropic map-germ arising from one-dimensional symplectic reduction
}

\author{
By GOO ISHIKAWA \\ Department of Mathematics, Hokkaido University, Sapporo 060, Japan
}

(Received 8 February 1991)

\section{Abstract}

In this paper, we classify generic isotropic map-germs arising from the symplectic reduction process relative to a hypersurface (i.e. one-dimensional reduction), up to symplectic equivalence in the $C^{\infty}$ category. These models are open Whitney umbrellas of arbitrary dimension and their suspensions. These singularities appear in the generalized Cauchy problem for Hamilton-Jacobi equations.

\section{Introduction}

A mapping from a manifold into a symplectic manifold is called isotropic if the pull-back of the symplectic form is zero. Isotropic mappings arise particularly in the process of (local) symplectic reduction due to Mardsen, Weinstein and Tulczyjew (see $[1,29,19])$.

Let $\left(M^{2(n+k}, \omega\right)$ be a symplectic manifold of dimension $2(n+k)$, where $\omega$ is the symplectic form, and $K^{2 n+k} \subset M$ be a coisotropic submanifold of codimension $k$. We denote by $(T K)^{\perp}$ the skew-orthogonal complement to $T K$ in $T M \mid K$ with respect to $\omega$. Remark that the rank of $(T K)^{\perp}$ is equal to $k$. Since $K$ is coisotropic, $(T K)^{\perp} \subset T K$ and $(T K)^{\perp}$ is integrable. We call $(T K)^{\perp}$ (resp. the induced foliation on $K$ ) the characteristic distribution (resp. the foliation) relative to $K$.

Ley $x \in K$. Then, in an open neighbourhood $U$ of $x$ in $K$, a submersion $\pi: U \rightarrow M^{\prime 2 n}$ is induced, where $M^{\prime}$ is the leaf space. Then $M^{\prime}$ has a unique symplectic structure $\omega^{\prime}$ up to symplectic diffeomorphisms of $M^{\prime}$ such that $\pi^{*} \omega^{\prime}=\omega \mid K$ (see [1]).

Let $N^{n}$ be an isotropic submanifold of $M^{2(n+k)}$ contained in $K^{2 n+k}$ and containing $x$. Then we see that $f=\pi \mid N: N, x \rightarrow M^{\prime}$ is isotropic, that is, $f^{*} \omega^{\prime}=0$, and that $f$ is immersive if and only if $T_{x} N \cap\left(T_{x} K\right)^{\perp}=0$. Such a germ $f$ is called an isotropic mapgerm arising from $k$-dimensional reduction.

In this paper, we concentrate on the case $k=1$, that is, the case when $K$ is a hypersurface. This situation arises in the generalized Cauchy problem for Hamilton-Jacobi equations $[1,15]$, where $M$ is regarded as the phase space, $K$ as an equation and $N$ as an initial condition, and we allow characteristic directions to be tangent to $N$. In this situation, the singularity of the isotropic map-germ obtained by reduction describes the tangency of characteristic directions to $N$ (and, therefore, the singularity of the extended Lagrangian variety from $N$ by characteristic curves).

Let $\left(M^{2 n+2}, \omega\right)$ be a $C^{\infty}$ symplectic manifold, $K^{2 n+1}$ be a $C^{\infty}$ hypersurface of $M$, and $N^{n}$ be an $n$-dimensional $C^{\infty}$ manifold. 
Denote by $\mathbf{I}=\mathbf{I}(N, K)$ the set of $C^{\infty}$ isotropic embeddings $i: N \rightarrow M$ with $i(N) \subset K$, endowed with the Whitney $C^{\infty}$ topology.

As local modes for singularities of isotropic mappings, we prepare isotropic mapgerms $f_{n, k}$. Consider the cotangent bundle $T^{*} \mathbb{R}^{n}$ with canonical coordinates $q_{1}, \ldots, q_{n}$; $p_{1}, \ldots, p_{n}$ and with the symplectic form $\omega=\sum_{i=1}^{n} d p_{i} \wedge d q_{i}$. Besides, consider the space $\mathbb{R}^{n}$ with coordinates $x_{1}, \ldots, x_{n}$. Then $f_{n, k}: \mathbb{R}^{n}, 0 \rightarrow T^{*} \mathbb{R}^{n},\left(0 \leqslant k \leqslant\left[\frac{1}{2} n\right]\right)$ is defined by

$$
\begin{gathered}
q_{i} \circ f_{n, k}=x_{i} \quad(1 \leqslant i \leqslant n-1), \\
u=q_{n} \circ f_{n, k}=\frac{x_{n}^{k+1}}{(k+1) !}+\sum_{i=1}^{k-1} x_{i} \frac{x_{n}^{k-i}}{(k-i) !}, \\
v=p_{n} \circ f_{n, k}=\sum_{i=0}^{k-1} x_{k+i} \frac{x^{k-i}}{(k-i) !},
\end{gathered}
$$

and

$$
p_{j} \circ f_{n, k}=\int_{0}^{x_{n}}\left(\frac{\partial v}{\partial x_{j}} \frac{\partial u}{\partial x_{n}}-\frac{\partial v}{\partial x_{n}} \frac{\partial u}{\partial x_{j}}\right) d x_{n} \quad(1 \leqslant j \leqslant n-1),
$$

that is,

$$
p_{j} \circ f_{n, k}= \begin{cases}-\sum_{i=0}^{k-1} x_{k+i} x_{n}^{2 k-i-j} /((k-i-1) !(k-j) !(2 k-i-j)) & (1 \leqslant j \leqslant k-1), \\ x_{n}^{3 k-j+1} /(k !(2 k-j) !(3 k-j+1)) & \\ \quad+\sum_{i=1}^{k-1} x_{i} x_{n}^{3 k-i-j} /((k-i-1) !(2 k-j) !(3 k-i-j)) & (k \leqslant j \leqslant 2 k-1), \\ 0 & (2 k \leqslant j \leqslant n) .\end{cases}
$$

We remark that each $f_{n, k}$ is a polynomial mapping of kernel rank one and of very simple form, and that it is a topological embedding.

We intend to classify isotropic map-germs under the following equivalence: In general, let $f: N^{n}, x \rightarrow\left(M^{2 n}, \omega\right)$ and $f^{\prime}: N^{\prime n}, x \rightarrow\left(M^{\prime 2 n}, \omega^{\prime}\right)$ be isotropic map-germs, where $\left(M^{2 n}, \omega\right)$ and $\left(M^{\prime 2 n}, \omega\right)$ are symplectic manifolds. We call $f$ symplectically equivalent to $f^{\prime}$ if there exist a diffeomorphism germ $\sigma: N, x \rightarrow N^{\prime}, x^{\prime}$ and a symplectic diffeomorphism germ $\tau: M, f(x) \rightarrow M^{\prime}, f^{\prime}\left(x^{\prime}\right),\left(\tau^{*} \omega^{\prime}=\omega\right)$, such that $\tau \circ f=f^{\prime} \circ \sigma$.

The purpose of this paper is to prove the following:

THeOREM 1. There exists an open dense subset $\mathbf{G}$ in $\mathbf{I}$ such that, for each $i \in \mathbf{G}$ and for each $x \in N$, the isotropic map-germ $f: N^{n}, x \rightarrow M^{\prime 2 n}$ arising from 1-dimensional reduction relative to $K$ is symplectically equivalent to some $f_{n, k}\left(0 \leqslant k \leqslant\left[\frac{1}{2} n\right]\right)$.

For $k=0, f_{n, 0}$ is just the zero-section $\zeta_{n}: \mathbb{R}^{n}, 0 \rightarrow T^{*} \mathbb{R}^{n}$ and is the model for Lagrangian immersions. We remark that any Lagrangian immersion-germs are symplectically equivalent to each other.

We can write $f_{n, k}=f_{2 k, k} \times \zeta_{n-2 k}$. Therefore $f_{n, k}$ is a suspension of $f_{2 k, k}$. We call $f_{2 n, n}$ the $2 n$-dimensional open Whitney umbrella. This is a fundamental object: however explicit investigations of it have only begun very recently.

Related to the problem of Lagrangian immersion of surfaces to four-dimensional symplectic manifolds, the (two-dimensional) open Whitney umbrella $f_{2,1}$ is introduced by Givental' $[14]$. The mapping $\left(f_{2,1}, e\right): \mathbb{R}^{2} \rightarrow T^{*} \mathbb{R}^{2} \times \mathbb{R}=J^{1}\left(\mathbb{R}^{2}, \mathbb{R}\right)$ defines a Legendrian variety, where $e$ is a generating function of $f_{2,1}$ (see Section 1). The wavefront set of this Legendrian variety is called the composed Whitney umbrella [14]. This object appears as a singularity of a tangent developable from a generic space curve $[8,25,27]$. It appears also in the study of relaxation equations with two slow variables [10]. 
Open Whitney umbrellas in higher dimension are described in [15]. In that paper, there are interesting characterizations of these singularities. For instance, the (image of a) $2 n$-dimensional open Whitney umbrella is symplectically equivalent to the total space of the conormal bundle of the $n$-dimensional open swallowtail in $\mathbb{R}^{2 n}$. (See also [4].) Furthermore, a similar result to Theorem 1 is proved in [15] for isotropic submanifolds belonging to an open dense subset in the space of all submanifolds of dimension $n$, but not in the space of isotropic submanifolds. (See [15], theorem 17, p. 104 and also [4], §5.6.) Therefore it was unfinished to determine the density. This gap is filled by Arnol'd in the case $n=2$ (unpublished). Thus our result can be regarded as the completion of the result of Givental' in any dimension. (This remark is based on a letter from Givental' to the author after the first draft of this paper.)

For further study of the singularities $f_{n, k}$, especially on their Maslov classes, see $[14,15,18]$.

A proof similar to the proof of Theorem 1 gives the following result:

THEOREM 2. There exists an open dense subset $\mathbf{G}^{\prime}$ in the space of $C^{\infty}$ isotropic mappings of kernel rank $\leqslant 1$ from an n-dimensional $C^{\infty}$ manifold $N$ to a $2 n$-dimensional $C^{\infty}$ symplectic manifold $M$ such that, for any $f \in \mathrm{G}^{\prime}$ and for any $x \in N$, the germ $f$ at $x$ is symplectically equivalent to some $f_{n, k}\left(0 \leqslant k \leqslant\left[\frac{1}{2} n\right]\right)$.

We should mention that, by Theorem 1 (resp. Theorem 2), for a generic isotropic map-germ arising from one-dimensional symplectic reduction (resp. of kernel rank one), the multiplicity merely as a map-germ turns to be the only invariant, under symplectic equivalence. (Notice that also the explicit normal forms are given in Theorems 1 and 2.)

In the usual singularity theory of $C^{\infty}$ mappings, generic map-germs are (topologically) classified by their local algebras (see $[22,13]$ for $C^{\infty}$-stable germs, and $[24,12]$ for $C^{0}$-stable germs). Therefore, it is very interesting to ask whether or not similar results hold for classification of generic isotropic map-germs arising from arbitrary $k$-dimensional reduction, in other words, for classification of generic isotropic map-germs of kernel rank $k$. In the singularity theory of mappings, the notion of determinacy is very powerful: see $[28,9]$. It is natural to apply this to the study of isotropic mappings. However, it is far from trivial and it seems particular to the case treated in this paper that the normal forms of isotropic map-germs are polynomials and that a jet of an isotropic map-germ has a polynomial representative (cf. Section 1). See [4], for other difficulties in generalizing our results.

We also remark that there are extensive studies, for instance in $[3,19]$, on the symplectic reduction (or push forward in the sense of Janeczko) of a Lagrangian manifold rather than an isotropic manifold. Though an open Whitney umbrella cannot obtain as a reduction of Lagrangian manifold, it arises as a component of a reduction of Lagrangian manifold: see $[4,17]$. This fact seems important for the classification of generic isotropic map-germs of kernel rank 1, under Lagrangian equivalence (Section 1). This classification problem is an interesting generalization of the Lagrangian singularity theory of Lagrangian immersions $[2,5]$. On this direction, we observe the following weak result:

THeOREM 3. For a generic isotropic mapping $f: N^{2 n} \rightarrow T^{*} \mathbb{R}^{2 n}$ of kernel rank $\leqslant 1$ and for a point $x \in N$, if the germ $f_{x}: N, x \rightarrow T^{*} \mathbb{R}^{2 n}$ has the maximal multiplicity, that is, $n+1$, then $f_{x}$ is Lagrange equivalent to $f_{2 n, n}$. 
Thus, the classification problem of germs of type $f_{n, k}$ under Lagrangian equivalence remains open in the case $n>2 k$.

In Section 1, we consider the relation between Lagrangian equivalence of isotropic map-germs and $R^{+}$-equivalence of generating functions. In Section 2 , we study the module of generating functions, in order to reduce generating functions to normal forms under $R^{+}$-equivalence. Theorem 1 is proved, in the last section, via a transversality argument. We also remark that Theorems 2 and 3 can be proved by a slight modification of the proof of Theorem 1.

Throughout this paper, all manifolds are assumed to be paracompact of class $C^{\infty}$ and mappings are also of class $C^{\infty}$.

\section{Generating functions and $R^{+}$-equivalence}

Let $g=\left(g_{1}, \ldots, g_{n}\right): N, x \rightarrow \mathbb{R}^{n}, 0$ be a map-germ, and $f: N, x \rightarrow T^{*} \mathbb{R}^{n}$ be an isotropic map-germ covering $g$, that is, $\pi \circ f=g$, where $\pi: T^{*} \mathbb{R}^{n} \rightarrow \mathbb{R}^{n}$ is the projection. Set $\theta=\sum_{i=1}^{n} p_{i} d q_{i}$ (Liouville form on $T^{*} \mathbb{R}^{n}$ ). Then $f^{*} \theta$ is closed. Thus, by Poincaré's lemma, $d e=f * \theta$ for some $e \in E_{N, x}$, where $E_{N, x}$ denotes the $\mathbb{R}$-algebra of functiongerms of $N, x$. The function-germ $e$ (which is unique up to an additive constant) is called a generating function of $f$.

Denote by $H_{g}$ the set of generating functions of isotropic map-germs covering $g$. The following relates $H_{g}$ with the 0th relative de Rham cohomology of $g$ (cf. [21]).

Lemma 1.1. $H_{g}=\left\{e \in E_{N, x} \mid d e \in\left\langle d g_{1}, \ldots, d g_{n}\right\rangle E_{N, x}\right\}$.

Proof. Let $e \in H_{g}$. Then $d e=f^{*} \theta$, for some isotropic $f: N, x \rightarrow T^{*} \mathbb{R}^{n}$. Then

$$
d e=\sum_{i=1}^{n} p_{i} \circ f d g_{i} .
$$

Conversely, suppose $d e=\sum_{i=1}^{n} a_{i} d g_{i}$, for some $a_{i} \in E_{N, x}$ covering $g$. Define $f$ by $p_{i} \circ f=a_{i}$ and $q_{i} \circ f=g_{i}(1 \leqslant i \leqslant n)$. Then $f$ is isotropic and covers $g$.

Definition 1.2. Two isotropic map-germs $f: N, x \rightarrow T * \mathbb{R}^{n}$ and $f^{\prime}: N^{\prime}, x^{\prime} \rightarrow T * \mathbb{R}^{n}$ are called Lagrange equivalent if there exist a diffeomorphism-germ $\sigma: N, x \rightarrow N^{\prime}, x^{\prime}$ and a $\pi$-fibre-preserving symplectic diffeomorphism-germ $T: T^{*} \mathbb{R}^{n}, f(x) \rightarrow T^{*} \mathbb{R}^{n}, f^{\prime}\left(x^{\prime}\right)$ such that $f^{\prime} \circ \sigma=T \circ f$.

If $f$ and $f^{\prime}$ are Lagrange equivalent, then they are symplectically equivalent.

The following notion is a variant of $R^{+}$-equivalence in [5], and it is utilized in [11].

Definition 1.3. Let $g: N, x \rightarrow \mathbb{R}^{n}, 0$ and $g^{\prime}: N^{\prime}, x^{\prime} \rightarrow \mathbb{R}^{n}, 0$ be map-germs and $e: N, x \rightarrow \mathbb{R}$ and $e^{\prime}: N^{\prime}, x^{\prime} \rightarrow \mathbb{R}$ be function-germs. Then two pairs $(e, g)$ and $\left(e^{\prime}, g^{\prime}\right)$ are called $R^{+}$equivalent if there exist diffeomorphism-germs $\sigma: N, x \rightarrow N^{\prime}, x^{\prime}$ and $\tau: \mathbb{R}^{n}, 0 \rightarrow \mathbb{R}^{n}, 0$, and a function-germ $\alpha: \mathbb{R}^{n}, 0 \rightarrow \mathbb{R}$ such that

$$
e=e^{\prime} \circ \sigma+\alpha \circ g \text { and } g^{\prime} \circ \sigma=\tau \circ g .
$$

Proposition 1 4. Let $f: N, x \rightarrow T^{*} \mathbb{R}^{n}$ and $f^{\prime}: N^{\prime}, x^{\prime} \rightarrow T^{*} \mathbb{R}^{n}$ be isotropic map-germs covering $g$ and $g^{\prime}$, with generating functions e and $e^{\prime}$ respectively. If $f$ and $f^{\prime}$ are Lagrange equivalent, then $(e, g)$ and $\left(e^{\prime}, g^{\prime}\right)$ are $R^{+}$-equivalent.

Furthermore, assume the set-germ of critical points of $g^{\prime}$ is nowhere dense. If $(e, g)$ and $\left(e^{\prime}, g^{\prime}\right)$ are $R^{+}$-equivalent, then $f$ and $f^{\prime}$ are Lagrange equivalent. 
Proof. First suppose $f$ and $f^{\prime}$ are Lagrange equivalent, and $(\sigma, T)$ realizes this equivalence. Write $T$ in the form

Then

$$
\begin{gathered}
(p, q) \mapsto(P(p, q), \tau(q)) . \\
\sum_{i=1}^{n} P_{i} d \tau_{i}-\sum_{i=1}^{n} p_{i} d q_{i}=d A(p, q),
\end{gathered}
$$

for some $A: T^{*} \mathbb{R}^{n}, f(x) \rightarrow \mathbb{R}$. Since $\partial A / \partial p_{i}=0(1 \leqslant i \leqslant n)$, we have $A=\pi^{*} \tilde{\alpha}$ for some $\tilde{\alpha}: \mathbb{R}^{n}, 0 \rightarrow \mathbb{R}$. Thus $T^{*} \theta=\theta+\pi^{*} d \tilde{\alpha}$.

Since $f^{\prime} \circ \sigma=T \circ f$, we see that

$$
d\left(\sigma^{*} e^{\prime}\right)=\sigma^{*} f^{\prime *} \theta=f^{*}\left(\theta+\pi^{*} d \tilde{\alpha}\right)=d\left(e+g^{*} \tilde{\alpha}\right) .
$$

Thus $e=e \circ \sigma-\tilde{\alpha} \circ g+c$ for some $c \in \mathbb{R}$. Then it suffices to set $\alpha=-\tilde{\alpha}+c$ to see that $(e, g)$ and $\left(e^{\prime}, g^{\prime}\right)$ are $R^{+}$-equivalent.

Next suppose that $(e, g)$ and $\left(e^{\prime}, g^{\prime}\right)$ are $R^{+}$-equivalent, and $(\sigma, \tau, \alpha)$ realizes this equivalence. Define a fibre-preserving symplectic diffeomorphism-germ $T: T^{*} \mathbb{R}^{n}$, $T_{0}^{*} \mathbb{R}^{n} \rightarrow T^{*} \mathbb{R}^{n}, T_{0}^{*} \mathbb{R}^{n}$ covering $\tau$ by $T^{*} \theta-\theta=-d \pi^{*} \alpha$. Then

$$
\left(T \circ f \circ \sigma^{-1}\right) * \theta=\sigma^{-1 * f *}\left(\theta-d \pi^{*} \alpha\right)=\sigma^{-1 *}\left(d e-d g^{*} \alpha\right)=\sigma^{-1 *}\left(d \sigma^{*} e^{\prime}\right)=d e^{\prime}=f^{\prime *} \theta .
$$

Both $T \circ f \circ \sigma^{-1}$ and $f^{\prime}$ cover $g^{\prime}$. Since the critical point set of $g^{\prime}$ is nowhere dense, as easily verified, each map-germ covering $g^{\prime}$ is uniquely determined by the pull-back of $\theta$. Hence $T \circ f \circ \sigma^{-1}=f^{\prime}$. Thus Proposition 1.4 is proved.

Now assume that $g$ is of the form $g=\left(x^{\prime}, u\left(x^{\prime}, x_{n}\right)\right): \mathbb{R}^{n}, 0 \rightarrow T^{*} \mathbb{R}^{n}$, for some coordinates $x_{1}, \ldots, x_{n}$ of the source, where $x^{\prime}=\left(x_{1}, \ldots, x_{n-1}\right)$. Let $f: \mathbb{R}^{n}, 0 \rightarrow T^{*} \mathbb{R}^{n}$ be an isotropic lifting of $g$. Set $v=p_{n} \circ f$. Then we have

Lemma 15. For a generating function e of $f$, there exists a function-germ $\beta=\beta\left(x^{\prime}\right)$ such that

$$
e\left(x^{\prime}, x_{n}\right)=\int_{0}^{x_{n}} v\left(x^{\prime}, t\right) \frac{\partial u}{\partial t}\left(x^{\prime}, t\right) d t+\beta\left(x^{\prime}\right) .
$$

Proof. Since $d e=\sum_{i=1}^{n-1} p_{i} \circ f d x_{i}+v d u$, we have $\partial e / \partial x_{n}=v\left(\partial u / \partial x_{n}\right)$. This leads the required result.

Given $v \in E_{n}$, with $v\left(x^{\prime}, 0\right)=0$, define $f_{v}: \mathbb{R}^{n}, 0 \rightarrow T^{*} \mathbb{R}^{n}$ by $\pi \circ f_{v}=g, p_{n} \circ f_{v}=v$ and

$$
p_{j} \circ f_{v}=\int_{0}^{x_{n}}\left\{\frac{\partial v}{\partial x_{j}}\left(x^{\prime}, t\right) \frac{\partial u}{\partial t}\left(x^{\prime}, t\right)-\frac{\partial v}{\partial t}\left(x^{\prime}, t\right) \frac{\partial u}{\partial x_{j}}\left(x^{\prime}, t\right)\right\} d t \quad(1 \leqslant j \leqslant n-1) .
$$

LeMMA 1.6. Let $g=\left(x^{\prime}, u\left(x^{\prime}, x_{n}\right)\right)$ and $v \in E_{n}$. (1) If $v\left(x^{\prime}, 0\right)=0$, then $f_{v}$ is an isotropic lifting of $g$. (2) Assume that the set-germ of critical points of $g$ is nowhere dense. Then any isotropic lifting $f$ of $g$ with $p_{n} \circ f=v$ is Lagrange equivalent to $f_{v-v\left(x^{\prime}, 0\right)}$.

Proof. (1) Set $e_{v}=\int_{0}^{x_{n}} v\left(x^{\prime}, t\right)(\partial u / \partial t)\left(x^{\prime}, t\right) d t$. Then we see that

$$
\partial e_{v} / \partial x_{j}=p_{j} \circ f_{v}+\left[v\left(x^{\prime}, t\right)\left(\partial u / \partial x_{j}\right)\left(x^{\prime}, t\right)\right]_{t=0}^{t=x_{n}} \quad(1 \leqslant j \leqslant n-1) .
$$

Since $v\left(x^{\prime}, 0\right)=0$, we have $d e_{v}=f_{v}^{*} \theta$. Hence we have (1).

(2) By Lemma 1.5, a generating function $e$ of $f$ is equal to

$$
e_{v-v\left(x^{\prime}, 0\right)}+v\left(x^{\prime}, 0\right) u\left(x^{\prime}, x_{n}\right)+\beta\left(x^{\prime}\right)
$$


for some $\beta$. Therefore, setting $\alpha=v\left(q_{1}, \ldots, q_{n-1}, 0\right) q_{n}+\beta\left(q_{1}, \ldots, q_{n-1}\right)$ in Definition 1·3, we see that $e$ and $e_{v-v\left(x^{\prime}, 0\right)}$ are $R^{+}$-equivalent. Thus, by Proposition 1.4, we have (2).

Example 1.7. Set $g=\left(x^{\prime}, u\left(x^{\prime}, x_{n}\right)\right)$, and $u=x_{n}^{k+1} /(k+1) !=\sum_{i=1}^{k-1} x_{i} x^{k-i} /(k-i) !$. Then $f_{n, k}=f_{v}$, where $v=\sum_{i=0}^{k-1} x_{k+i} x_{n}^{k-i} /(k-i)$ !.

Remark 1.8. Using Lemma $1 \cdot 6$, we can construct a perturbation of $f$ in the space of isotropic mappings, from perturbations of $u=q_{n} \circ f$ and $v=p_{n} \circ f$.

\section{Generators of the module of generating functions}

Consider the space $\mathbb{R}^{n}=\mathbb{R}^{n-1} \times \mathbb{R}$ with coordinates $y_{1}, \ldots, y_{n-1} ; t$. Set

$$
\mathbb{R}^{r}=\left\{\left(y_{1}, \ldots, y_{n-1}\right) \in \mathbb{R}^{n-1} \mid y_{r+1}=\ldots=y_{n-1}=0\right\} \quad(0 \leqslant r \leqslant n-1) .
$$

Denote by $j_{r}: \mathbb{R}^{r-1} \times \mathbb{R} \rightarrow \mathbb{R}^{r} \times \mathbb{R}$ the inclusion defined by

$$
j_{r}\left(y_{1}, \ldots, y_{r-1} ; t\right)=\left(y_{1}, \ldots, y_{r-1}, 0 ; t\right) \quad(1 \leqslant r \leqslant n-1) .
$$

Then, set $\phi_{r}=j_{n-1} \circ \ldots \circ j_{r+1}: \mathbb{R}^{r} \times \mathbb{R} \rightarrow \mathbb{R}^{n-1} \times \mathbb{R}(0 \leqslant r<n-1)$, and $\phi_{n-1}$ the identity on $\mathbb{R}^{n-1} \times \mathbb{R}$.

Denote by $E_{r+1}$ the $\mathbb{R}$-algebra of $C^{\infty}$ function-germs at $(0,0)$ in $\mathbb{R}^{r} \times \mathbb{R}$. Then $E_{r+1}$ has unique maximal ideal $m_{r+1}$ generated by $y_{1}, \ldots, y_{r}$ and $t$.

Now fix an element of $E_{n}$,

$$
P=t^{k} / k !+a_{1}(y) t^{k-1} /(k-1) !+\ldots+a_{k}(y),
$$

which is a polynomial with respect to $t$, and assume that $a_{i}$ is an analytic map-germ with $a_{i}(0)=0(1 \leqslant i \leqslant k)$. Then set

$$
\begin{gathered}
B_{r}=\left\{h \in E_{r+1} \mid \partial h / \partial t \text { is a multiple of } \phi_{r}^{*} P \text { in } E_{r+1}\right\} \quad(0 \leqslant r \leqslant n-1), \\
H_{l}(y, t)=\int_{0}^{t} \frac{s^{l}}{l !} P(y, s) d s \in E_{n+1} \quad(0 \leqslant l \leqslant k),
\end{gathered}
$$

and

$$
\Gamma_{r}=\left(y_{1}, \ldots, y_{r}, \phi_{r}^{*} H_{0}\right): \mathbb{R}^{r} \times \mathbb{R} \rightarrow \mathbb{R}^{r} \times \mathbb{R} \quad(0 \leqslant r \leqslant n-1) .
$$

Remark 2.1. Set $g=\Gamma_{n-1}=\left(y, t^{k+1} /(k+1) !+a_{1}(y) t^{k} / k !+\ldots+a_{k}(y) t\right)$. Then $H_{g}=$ $B_{n-1}$ in $E_{n}$.

To see the structure of $B_{n-1}$, we show the following:

Proposition 2 2. $\left(a_{r}\right) 1, \phi_{r}^{*} H_{1}, \ldots, \phi_{r}^{*} H_{k}$ generate $B_{r}$ over $E_{r+1}$ via $\Gamma_{r}^{*}: E_{r+1} \rightarrow E_{r+1}$ $(0 \leqslant r \leqslant n-1) .\left(b_{r}\right) \operatorname{Ker} j_{r+1}^{*} \subset \Gamma_{r+1}^{*} m_{r+1+1} B_{r+1}$, where $j_{r+1}^{*}: B_{r+1} \rightarrow B_{r}(0 \leqslant r<n-1)$. $\left(c_{r}\right) j_{r+1}^{*}$ induces an isomorphism

$$
j: B_{r+1} / \Gamma_{r+1}^{*} m_{r+1+1} B_{r+1} \cong B_{r} / \Gamma_{r}^{*} m_{r+1} B_{r}
$$

of $\mathbb{R}$-vector spaces $(0 \leqslant r<n-1)$.

Proof. Step 1: $\left(a_{0}\right)$. It is easy to see that

$$
B_{0}=\left\{h(t) \in E_{1} \mid \partial h / \partial t \text { is a multiple of } t^{k}\right\}
$$

is generated by $1, t^{k+2}, \ldots, t^{2 k+1}$ over $E_{0+1}$ via $\left(t^{k+1}\right)^{*}: E_{0+1} \rightarrow E_{0+1}$. Thus $B_{0}$ is generated by $1, \phi_{0}^{*} H_{1}, \ldots, \phi_{0}^{*} H_{k}$ over $E_{0+1}$ via $\Gamma_{0}^{*}: E_{0+1} \rightarrow E_{0+1}$. Therefore we have $\left(a_{0}\right)$. 
Step 2: $\left(b_{r}\right),(0 \leqslant r<n-1)$. Let $h\left(y_{1}, \ldots, y_{r}, 0 ; t\right)=0$. Then $h=y_{r+1} g\left(y_{1}, \ldots, y_{r+1}, t\right)$ for some $g \in E_{r+1+1}$ and $\partial h / \partial t=y_{r+1}(\partial g / \partial t)$. On the other hand, $\partial h / \partial t=w \phi_{r+1}^{*} P$ for some $w \in E_{r+1+1}$. Set $y_{r+1}=0$. Then $w\left(y_{1}, \ldots, y_{r}, 0, t\right) \phi_{r}^{*} P=0$. Since $\phi_{r}^{*} P$ is not flat, we see that $w\left(y_{1}, \ldots, y_{r}, 0, t\right)=0$. Thus $w=y_{r+1} \tilde{w}$ for some $\tilde{w} \in E_{r+1+1}$. Hence $y_{r+1}\left(\partial g / \partial t-\tilde{w} \phi_{r+1}^{*} P\right)=0$. Therefore $\partial g / \partial t=\tilde{w} \phi_{r+1}^{*} P$. This implies that $g \in B_{r+1}$ and $h \in \Gamma_{r+1}^{*} m_{r+1+1} B_{r+1}$. We have $\left(b_{r}\right),(0 \leqslant r<n-1)$.

Step 3: $\left(a_{r}\right)$ and $\left(b_{r}\right)$ imply $\left(c_{r}\right),(0 \leqslant r<n-1)$. For this, first we will show that $j_{r+1}^{*}$ is surjective. In fact, let $h \in B_{r}$. By $\left(a_{r}\right)$,

$$
h=\Gamma_{r}^{*} w_{0}+\sum_{s=1}^{k} \Gamma_{r}^{*} w_{s} \phi_{r}^{*} H_{s}
$$

for some $w_{0}, w_{1}, \ldots, w_{k} \in E_{r+1}$. Set

$$
\tilde{h}=\Gamma_{r+1}^{*} \tilde{w}_{0}+\sum_{s=1}^{k} \Gamma_{r+1}^{*} \tilde{w}_{s} \phi_{r+1}^{*} H_{s}
$$

where $\tilde{w}_{s} \in E_{r+1+1}$ is defined by $\tilde{w}_{s}\left(y_{1}, \ldots, y_{r}, y_{r+1} ; t\right)=w_{s}\left(y_{1}, \ldots, y_{r} ; t\right)$. Then $j_{r+1}^{*} \tilde{h}=h$. Therefore $j$ is surjective.

Next we will show that $j$ is injective. For this, let $h \in B_{r+1}$ with $j_{r+1}^{*} h \in \Gamma_{r}^{*} m_{r+1} B_{r}$. Then $j_{r+1}^{*} h=\sum_{s} \Gamma_{r}^{*} w_{s} \cdot u_{s}$ (with $\left.u_{s} \in B_{r}, w_{s} \in m_{r+1}\right)$. By $\left(a_{r}\right)$, there exists $\tilde{u}_{2} \in B_{r+1}$ such that $j_{r+1}^{*} \tilde{u}_{s}=u_{s}$. Set

$$
\tilde{h}=\sum_{s} \Gamma_{r+1}^{*} \tilde{w}_{s} \tilde{u}_{2} \in \Gamma_{r+1}^{*} m_{r+1+1} B_{r+1} .
$$

Then $j_{r+1}^{*} \tilde{h}=j_{r+1}^{*} h$. Thus $j_{r+1}^{*}(h-\tilde{h})=0$, and $h-\tilde{h} \in \operatorname{Ker} j_{r+1}^{*}$. By $\left(b_{r}\right)$,

Hence $h \in \Gamma_{r+1}^{*} m_{r+1+1} B_{r+1}$.

$$
h-\tilde{h} \in \Gamma_{r+1}^{*} m_{r+1+1} B_{r+1} \text {. }
$$

Step 4: $\left(a_{r}\right)$ and $\left(c_{r}\right)$ imply $\left(a_{r+1}\right),(0 \leqslant r<n-1)$. We remark that

$$
j_{r+1}^{*} \phi_{r+1}^{*} H_{l}=\left(\phi_{r+1} \circ j_{r+1}\right) * H_{l}=\phi_{r}^{*} H_{l} \quad(1 \leqslant l \leqslant k) .
$$

By $\left(a_{r}\right), 1, \phi_{r}^{*} H_{1}, \ldots, \phi_{r}^{*} H_{k}$ generate $B_{r} / \Gamma_{r} m_{r+1} B_{r}$ over $\mathbb{R}$. Thus $1, \phi_{r+1}^{*} H_{1}, \ldots, \phi_{r+1}^{*} H_{k}$ generate $B_{r+1} / \Gamma_{r+1}^{*} m_{r+1+1} B_{r+1}$ over $\mathbb{R}$, by $\left(c_{r}\right)$.

By [16], we see $B_{r+1}$ is a differentiable algebra in the sense of Malgrange. Then, we have $\left(a_{r+1}\right)$ by Malgrange's preparation theorem for differentiable algebras ([20]).

\section{Proof of theorems}

Let $\mathbf{I}$ be as in Theorem 1 and let $i \in \mathbf{I}$. Then there exist a locally finite open covering $\left\{V_{\lambda}\right\}_{\lambda \in \Lambda}$ of $N$ and a collection of charts $\left(U_{\lambda} ; P_{0}, \ldots, P_{n}, Q_{0}, \ldots, Q_{n}\right)$ of $M$ such that $i\left(V_{\lambda}\right) \subset$ $U_{\lambda}, K \cap U_{\lambda}=\left\{P_{0}=0\right\}, \omega \mid U_{\lambda}=\sum_{j=0}^{n} d P_{j} \wedge d Q_{j}$ and $\left(Q_{1}, \ldots, Q_{n-1}\right) \circ i: V_{\lambda} \rightarrow \mathbb{R}^{n-1}$ is a submersion. In this setting, for each $\lambda$, the reduction $f$ is written as $\left(P_{1}, \ldots, P_{n}, Q_{1}, \ldots\right.$, $\left.Q_{n}\right) \circ i: V_{\lambda} \rightarrow T^{*} \mathbb{R}^{n}$.

As the generic condition, we impose that the mapping

$$
f^{\prime}=\left(Q_{1} \circ i, \ldots, Q_{n} \circ i, P_{n} \circ i\right): V_{\lambda} \rightarrow \mathbb{R}^{n-1}
$$

is of Morin type [26]. More precisely, we define $i \in \mathrm{G}$ if and only if the $r$-jet extension $j^{r} f^{\prime}: V \rightarrow J^{r}\left(V, \mathbb{R}^{n+1}\right)$ of $f^{\prime}$ is transverse to $\sum^{1_{k}, 0}$ for $0 \leqslant k \leqslant r$, where $r=\left[\frac{1}{2} n\right]+2$ and $\sum^{1_{k}, 0}=\Sigma^{1, \ldots, 1,0},(k$ times $)$, is the Thom-Boardman symbol: see $[6,23]$. 
The transversality condition for $f^{\prime}$ is achieved by small perturbations of $Q_{n} \circ i$ and $P_{n} \circ i$. These perturbations induce an isotropic perturbation of $f$, therefore, of $i \mid V_{\lambda}$, fixing $Q_{0} \circ i$. (Notice also that the condition is open, because of Whitney regularity of the stratification.) Hence, we see that $\mathbf{G}$ is open dense in $\mathbf{I}$. We remark that the codimension of $\Sigma^{1_{k}, 0}$ is equal to $2 k$, and $\left(j^{r} f^{\prime}\right)^{-1}\left(\Sigma^{1_{k}, 0}\right)=\varnothing$ if $n>2 k$.

Now let $i \in \mathbf{G}$ and $x^{0} \in N$. Assume $x^{0} \in V_{\lambda}$ and $x^{0} \in\left(j^{r} f^{\prime}\right)^{-1}\left(\Sigma^{1_{k}, 0}\right)$. Consider the reduction $f: N, x^{0} \rightarrow T^{*} \mathbb{R}^{n}$. Then $f$ is symplectically equivalent to an isotropic mapgerm $\tilde{f}: \mathbb{R}^{n}, 0 \rightarrow T * \mathbb{R}^{n}$ with

$$
\begin{aligned}
& q_{j} \circ \tilde{f}=x_{j} \quad(1 \leqslant j \leqslant n-1), \\
& q_{n} \circ \tilde{f}=u\left(x^{\prime}, t\right)=t^{k+1} /(k+1) !+\sum_{j=1}^{k-1} x_{j} t^{k-j} /(k-j) !, \\
& p_{n} \circ \tilde{f}=v\left(x^{\prime}, t\right),
\end{aligned}
$$

with $(\partial v / \partial t)(0,0)=\ldots=\left(\partial^{k} v / \partial t^{k}\right)(0,0)=0$, for some coordinate $\left(x^{\prime}, t\right)$ of $\mathbb{R}^{n}, 0$, where $x^{\prime}=\left(x_{1}, \ldots, x_{n-1}\right)$.

In fact, first set $q_{i}=Q_{i}-Q_{i}\left(x^{0}\right), p_{i}=P_{i}-P_{i}\left(x^{0}\right)$ and $x^{\prime}=\left(q_{1} \circ i, \ldots, q_{n-1} \circ i\right)$. Then $\left(x^{\prime}, q_{n} \circ i, p_{n} \circ i\right): N, x^{0} \rightarrow \mathbb{R}^{n+1}$ is of Morin type. Second, we may assume the order of $q_{n} \circ i \mid\left\{x^{\prime}=0\right\}$ is not greater than that of $p_{n} \circ i \mid\left\{x^{\prime}=0\right\}$, and it is equal to $k+1$, changing $q_{n}$ and $p_{n}$ if necessary. Then $q_{n} \circ i$ is an universal deformation of $q_{n} \circ i \mid\left\{x^{\prime}=0\right\}$ : see [7]. Lastly, it suffices to use the symplectic diffeomorphism of $T^{*} \mathbb{R}^{n}$, which is the natural lift of a diffeomorphism of type $\left(q^{\prime}, q_{n}\right) \mapsto\left(\phi\left(q^{\prime}\right), \pm q_{n}+\beta\left(q^{\prime}\right)\right)$, where $q^{\prime}=\left(q_{1}, \ldots, q_{n-1}\right)$, and a diffeomorphism of source.

Let $e: \mathbb{R}^{n}, 0 \rightarrow \mathbb{R}$ be a generating function of $\tilde{f}$. Then

$$
d e=\sum_{i=1}^{n-1} p_{j} \circ \tilde{f} d x_{i}+v d u .
$$

Hence $\partial e / \partial t=v(\partial u / \partial t)$. Thus $e$ belongs to $B_{n-1}$ introduced in Section 2, setting $P=\partial u / \partial t$, with respect to the coordinate $(y, t)=\left(x^{\prime}, t\right)$. We remark that, in this situation, $H_{0}=u$ and $\Gamma_{n-1}=\left(x_{1}, \ldots, x_{n-1}, u\right)$ is identified with $g=\left(q_{1} \circ \tilde{f}, \ldots, q_{n} \circ \tilde{f}\right)$ : $\mathbb{R}^{n}, 0 \rightarrow \mathbb{R}^{n}, 0$.

By $\left(a_{n-1}\right)$ of Proposition 2.2, we have $e=A_{0} \circ g+\sum_{j=1}^{k} A_{j} \circ g H_{j}$, for some functiongerms $A_{0}, A_{1}, \ldots, A_{k}: \mathbb{R}^{n}, 0 \rightarrow \mathbb{R}$. Then we see that

$$
v=\left(\partial A_{0} / \partial q_{n}\right) \circ g+\sum_{j=1}^{n}\left(\partial A_{j} / \partial q_{n}\right) \circ g H_{j}+\sum_{j=1}^{k} A_{j} \circ g t^{j} / j ! .
$$

In particular, by the definition of $H_{j}(1 \leqslant j \leqslant k)$, we see that $\partial^{j} v / \partial t^{j} \equiv A_{j} \circ g$, modulo the ideal $\left\langle x_{1}, \ldots, x_{k-1}, t\right\rangle=\left\langle\partial u / \partial t, \ldots, \partial^{k} u / \partial t^{k}\right\rangle$ in $E_{n}$.

From the condition $(\partial v / \partial t)(0,0)=\ldots=\left(\partial^{k} v / \partial t^{k}\right)(0,0)=0$, we have $A_{1}(0)=\ldots=$ $A_{k}(0)=0$. By the transversality condition, $\left(\partial u / \partial t, \ldots, \partial^{k} u / \partial t^{k}, \partial v / \partial t, \ldots, \partial^{k} v / \partial t^{k}\right)$ is a submersion at 0 in $\mathbb{R}^{n}$. Hence we have

$$
d q_{1} \wedge \ldots \wedge d q_{k-1} \wedge d A_{1} \wedge \ldots \wedge d A_{k} \wedge d q_{n} \neq 0
$$

at 0 in $\mathbb{R}^{n}$. Define $\sigma: \mathbb{R}^{n}, 0 \rightarrow \mathbb{R}^{n}, 0$ by

$$
x_{i} \circ \sigma= \begin{cases}x_{i} & (1 \leqslant i \leqslant k-1,2 k \leqslant i \leqslant n), \\ A_{2 k-i} \circ g & (k \leqslant i \leqslant 2 k-1) .\end{cases}
$$


Then $H_{j} \circ \sigma=H_{j} \quad(1 \leqslant j \leqslant k)$, and $g \circ \sigma=\tau \circ g$, for some diffeomorphism-germ $\tau: \mathbb{R}^{n}, 0 \rightarrow \mathbb{R}^{n}, 0$. Furthermore,

$$
\begin{gathered}
e=A_{0} \circ g+\sum_{j=1}^{k} A_{j} \circ g H_{j}=A_{0} \circ g+e^{\prime} \circ \sigma, \\
e^{\prime}=\sum_{j=1}^{k} x_{2 k-j} H_{j}=\int_{0}^{t}\left\{\sum_{j=1}^{k} x_{2 k-j} s^{j} / j !\right\}(\partial u / \partial s)\left(x^{\prime}, s\right) d s
\end{gathered}
$$

where

is a generating function of $f_{n, k}$. Thus $(e, g)$ and $\left(e^{\prime}, g\right)$ are $R^{+}$-equivalent, and $\tilde{f}$ is Lagrange, hence symplectically, equivalent to $f_{n, k}$ by Proposition 1.4. This completes the proof of Theorem 1 .

To prove Theorem 2, it is sufficient to start with $f$, instead of $i$, in the proof of Theorem 1.

To see Theorem 3, we remark that, for a generic isotropic mapping $f: N^{2 n} \rightarrow T^{*} \mathbb{R}^{2 n}$ of kernel rank $\leqslant 1$, each point, where the multiplicity of $f$ is $n+1$, is isolated. Thus it is sufficient to add the condition that, at each such point $x, \pi \circ f: N, x \rightarrow \mathbb{R}^{2 n}$ is of kernel rank one of multiplicity $n+1$. Since we use only Lagrangian equivalences in the proof of Theorem 1, except for exchanging $q_{n}$ and $p_{n}$, and this exchanging is not necessary in this case, we see $f_{x}$ is Lagrange equivalent to $f_{2 n, n}$.

The author would like to thank Professor A. B. Givental' and Professor S. Izumiya for valuable comments and information.

\section{REFERENCES}

[1] R. Abraham and J. E. Marsden. Foundation of Mechanics, 2nd edn. (Benjamin, 1978).

[2] V. I. ARNOL'D. Normal forms for functions near degenerate critical points, the Weyl groups of $A_{k}, D_{k}, E_{k}$ and Lagrangian singularities. Funktsional Anal. i Prilozhen. 6 (1972), 3-25.

[3] V. I. ARNOL'D. Singularities in variational calculus. J. Soviet Math. 27 (1984), 2679-2713.

[4] V. I. ARnol'D. Singularities of Caustics and Wave Fronts (Kluwer Academic Publishers, 1990).

[5] V. I. ARNOL'D, S. M. GUSEIN-ZADE and A. N. VARChEnKo. Singularities of Differentiable Maps I (Birkhäuser, 1985).

[6] M. Boardman. Singularities of differentiable maps. Inst. Hautes Études Sci. Publ. Math. 33 (1967), 21-57.

[7] Th. Bröcker. Differentiable Germs and Catastrophes. London Math. Soc. Lecture Note Ser. no. 17 (Cambridge University Press, 1975).

[8] J. CLEAVE. The form of the tangent developable at points of zero torsion on space curves. Math. Proc. Cambridge Philos. Soc. 88 (1980), 403-407.

[9] J. Damon. The Unfolding and Determinacy Theorems for Subgroups of $\mathscr{A}$ and $\mathscr{K}$. Memoirs Amer. Math. Soc. no. 50 (American Mathematical Society, 1984).

[10] A. A. DavyDov. The normal form of slow motions of an equation of relaxation type and fibrations of binormal surfaces. Math. USSR-Sb. 60 (1988), 133-141.

[11] J. P. Dufour. Familles de courbes planes différentiables. Topology 22 (1983), 449-474. [12] M. FukUDA and T. FuKUDA. Algebra $Q(f)$ determine the topological types of generic map
germs. Invent. Math. 51 (1979), 231-237.

[13] C. G. Grbson. Singular Points of Smooth Mappings (Pitman, 1979).

[14] A. B. GiventaL'. Lagrangian imbeddings of surfaces and unfolded Whitney umbrella. Funktsional Anal. i Prilozhen. 20 (1986), 35-41.

[15] A. B. GiventaL'. Singular Lagrangian varieties and their Lagrangian mappings. In Contemporary Problems of Mathematics, Itogi Nauki i Tekhniki, Ser. Sovrem. Probl. Mat. 33, (Vsesoyuz, Inst. Naukn. i Tekhn. Inform., 1988), pp. 55-112. [16] G. Ishrkawa. Families of functions dominated by distributions of $C$-classes of mappings.
Ann. Inst. Fourier (Grenoble) 33 (1983), 199-217.

[17] G. Ishikawa. Parametrization of a singular Lagrangian variety. Trans. Amer. Math. Soc., to appear. 
[18] G. IsHikawA. Maslov class of an isotropic map-germ arising from one dimensional symplectic reduction. (Preprint.)

[19] S. JANECZKO. Generating families for images of Lagrangian submanifolds and open swallowtails. Math. Proc. Cambridge Philos. Soc. 100 (1986), 91-107.

[20] B. Malgrange. Ideals of Differentiable Functions. (Oxford University Press, 1966).

[21] B. Malgrange. Frobenius avec singularités, 2. Le cas general. Invent. Math. 39 (1977), 67-89.

[22] J. N. MATHER. Stability of $C^{\infty}$ mappings IV: Classification of stable germs by $\mathbb{R}$ algebras. Inst. Hautes Études Sci. Publ. Math. 37 (1969), 223-248.

[23] J. N. Mather. On Thom-Boardman singularities. In Dynamical Systems (ed. M. M. Peixoto) (Academic Press, 1973), pp. 233-248.

[24] J. N. Mather. How to stratify mappings and jet spaces. In Singularités d'Applications Différentiables, Lecture Notes in Math. vol. 535 (Springer-Verlag, 1976), pp. 128-176.

[25] D. Mond. Singularities of the tangent developable surface of a space curve. Quart. J. Math. Oxford Ser. (2) 40 (1989), 79-91.

[26] B. Morin. Formes canonique des singularités d'une application différentiable, C.R. Acad. Sci. Paris 260 (1965), 5662-5665.

[27] O. P. Sherbak. Projectively dual curves and Legendre singularities. Selecta Math. Soviet 5 (1986), 391-421.

[28] C. T. C. WALL. Finite determinacy of smooth map-germs. Bull. London Math. Soc. 13 (1981), 481-539.

[29] A. Weinstern. Lectures on Symplectic Manifolds. Regional Conf. Series in Math. no. 29 (American Mathematical Society, 1977). 